\title{
Neisseria elongata sp.nov., a Rod-shaped Member of the Genus Neisseria. Re-evaluation of Cell Shape as a Criterion in Classification
}

\author{
By K. BØVRE AND E. HOLTEN \\ Kaptein W. Wilhelmsen og Frues Bakteriologiske Institutt, University of Oslo, \\ Rikshospitalet, Oslo, Norway \\ (Accepted for publication 6 October 1969)
}

\begin{abstract}
SUMMARY
A rod-shaped, non-motile, Gram-negative, oxidase-positive and asaccharolytic organism found in the human nasopharynx is allotted to genus Neisseria and named Neisseria elongata. The strain M 2, which is the only isolate, is proposed as the type strain. The average guanine + cytosine $(\mathrm{G}+\mathrm{C})$ content of its DNA is 53 mole \%. Genetic transformation of streptomycin resistance reveals a comparatively high compatibility with $N$. meningitidis and a strain designated $N$. flava, with ratios of interstrain to autologous transformation frequency in the range $0.0 \mathrm{I}$ to $0 . \mathrm{I}$. On the other hand, there is no affinity in streptomycin-resistance transformation between $N$. elongata and members of genus Moraxella, including the old concepts $N$. catarrhalis and $N$. ovis. The family Neisseriaceae now appears to consist of two genera, Neisseria and Moraxella, each containing both coccal and rod-shaped species, which makes cell shape questionable as a highly weighted criterion in the construction of bacterial genera and higher taxa.
\end{abstract}

\section{INTRODUCTION}

Previous investigations by means of genetic transformation and determination of DNA base composition (Bøvre, I967 b; Bøvre, Fiandt \& Szybalski, 1969) revealed strong indications of relationships between rod-shaped moraxellas and a group of coccal species within the family Neisseriaceae (Neisseria catarrhalis, N. caviae, N. ovis). This observation, which was later corroborated by RNA-to-DNA hybridization (K. Bøvre, unpublished), led Henriksen \& Bøvre (I968) to propose that these 'false neisserias' (Baumann, Doudoroff \& Stanier, I968) be grouped together with the rodshaped moraxellas in the genus Moraxella, proposed to belong in Neisseriaceae. The revised genus Moraxella would then consist of both rod-shaped and coccal species, with guanine + cytosine $(G+C)$ contents of their DNA in the range 40 to 45 mole $\%$. The remaining species of the family, which had $\mathrm{G}+\mathrm{C}$ contents in the neighbourhood of 50 mole $\%$ and which were interrelated in terms of genetic transformation (Catlin \& Cunningham, I96r ; Bøvre, I965, I967 b) and isolated from rodshaped moraxellas and 'false neisserias' in these terms (Catlin \& Cunningham, I96I; Bøvre, I965, 1967a,b), were retained in the genus Neisseria ('true neisserias'). The genus Neisseria seemed at the time to consist exclusively of coccal organisms: $N$. gonorrhoeae, N. meningitidis, N. sicca, N. subflava ( N. flava, N. perflava, N. subflava), $N$. flavescens, $N$. cinerea (Henriksen \& Bøvre, 1968). The present report provides evidence that this genus also contains a rod-shaped species. 


\section{METHODS}

Isolation. The rod-shaped bacterial strain M 2 was isolated from the nasopharynx of a presumably healthy person in northern Norway. It evoked interest initially because a few streptomycin-resistant transformants were elicited in the strain by DNA of Neisseria meningitidis. This finding was a result of a screening for competence of transformation (undertaken by E. H.) of oxidase-positive strains isolated from the upper respiratory tract of military personnel.

Morphological, cultural and biochemical tests. With the modifications mentioned below, these were identical with the procedures previously described (Bøvre \& Henriksen, $1967 b$ ). Heat resistance was studied in a water bath at $45^{\circ}$ and $56^{\circ}$. The amino acids + biotin medium used was as described by Baumann et al. (1968). The basal medium used for study of growth on different carbon sources was identical with the mixture of salts used in a defined fluid medium for meningococci by Jyssum (I965); all organic substances were omitted. Ammonium chloride was the sole source of nitrogen. Before use, glucose, maltose, acetate, citrate or lactate was added from a sterile $10 \%(\mathrm{w} / \mathrm{v})$ solution to a final concentration of $\mathrm{I} \%$. The inoculum for the two last-mentioned media consisted of $0.0 \mathrm{I} \mathrm{ml}$. from a $20-\mathrm{hr}$ broth culture in $10 \mathrm{ml}$. medium. The cultures were observed for 3 days of incubation with continuous shaking. Apparent growth was checked by subculture in the same medium. Acid production from carbohydrates was tested for in Mueller-Hinton broth with I \% glucose, maltose, sucrose, lactose, fructose, arabinose, galactose, xylose or mannitol, respectively, and with phenol red as indicator (Beno, Devine \& Larson, I968). Cultures were incubated for 4 weeks, and examined daily during the first 10 days. When otherwise not specified, incubation was at $37^{\circ}$ and in most cases also at $33^{\circ}$.

Buoyant density determination of DNA. Preparation of DNA and analytical $\mathrm{CsCl}$ density gradient centrifugation were done according to methods previously described (Bøvre et al. 1969).

Transformation methods. In addition to the strain M2, the following strains were used either as source of transforming mutant DNA or as recipients in the experiments designed to characterize the genetic relations of the new isolate in a quantitative way: Neisseria meningitidis в 8 I 52/66; N. meningitidis м6; N. flava АTCC I422 I; Moraxella nonliquefaciens $4663 / 62 ; M$. nonliquefaciens NCTC $7784 ; M$. nonliquefaciens $6121 / 68$; $M$. osloenisis A 1920; $M$. phenylpyrouvica 2863; M. kingii 4177/66; N. catarrhalis NE I I $N$. ovis I99/55. The strains (except $N$. flava ATCC I422I) have previously been characterized in intraspecies and interspecies transformation studies, which have been extensively referred to recently (Baumann et al. I968; Bøvre, I967b; Bøvre et al. 1969). The asaccharolytic $N$. meningitidis strain в 8I52/66 was studied in transformation a short time ago (Bøvre, 1969), as was also the strain $M$. nonliquefaciens $612 \mathrm{I} / 68$ (Henriksen \& Bøvre, I969). Most of the strains have also been characterized in terms of DNA base composition (Bøvre et al. 1969). The representativeness of the strains as regards their respective species designations is therefore well established. The old designation 'Neisseria' for the 'catarrhalis' and 'ovis' strains is used throughout this paper instead of 'Moraxella', which according to Henriksen \& Bøvre (I968) is the correct genus for these species. This is done because these designations were used in the papers referred to. We also use the name $N$. flava, which probably should be included in $N$. subflava, as deduced by Henriksen \& Bøvre from the transformation 
results of Catlin \& Cunningham (I96I). Neisseria flava ATCC I422 I was found to produce yellow pigment on coagulated serum medium and to produce acid from glucose, maltose and fructose, but not from sucrose or lactose.

The procedure for screening of competence (see section on isolation), which revealed that strain M 2 was possibly a unique rod-shaped organism as regards genetic relations, was described by Jyssum \& Lie (I965). It was done by the streptomycin underlayering technique introduced by Bøvre \& Henriksen (1962) and described further by Bøvre (1964). The principles of mutant selection, preparation of transforming DNA and transformation techniques have been described in connexion with application of the methods for moraxellas and neisserias (Bøvre, 1964, I967a,b). The genetic marker used was exclusively one-step mutation to high streptomycin resistance (> $1000 \mu \mathrm{g}$. streptomycin $/ \mathrm{ml}$.). Briefly, two principal methods were used in the present study: (I) a strictly quantitative procedure with short-term (20 min.) DNA exposure, discontinued by means of DNase; (2) a more sensitive but less accurate (semiquantitative) procedure where the DNA exposure was continued (DNase treatment omitted) after plating the fluid DNA + recipient mixture. In the latter modification, the increasing number of colonies containing transformants was limited by the addition of streptomycin by the underlayering technique at the end of the period allowed for phenotypic expression of transformants (after 5 to $7 \mathrm{hr}$ incubation). The only modification of the previous methods was that the recipients of strains M $2, N$. meningitidis B 8I 52/66 and $N$. flava ATCC I422I, were stored for 20 to $30 \mathrm{~min}$. at $+4^{\circ}$ before use in transformation, instead of at $-20^{\circ}$. The quantitative transformation procedure was done with bacteria in the lag phase. A few other experiments indicated that results obtained with lag-phase cultures were of the same order as with bacteria in the exponential phase of growth.

\section{RESULTS}

\section{Strain $M 2$}

Morphological, cultural and biochemical characters. Microscopically the strain M 2 appeared as short and slender rods, often arranged as diplobacilli or in short chains. The length of the organisms was not always uniform in the same preparation, some of the organisms being almost coccoid. However, stained smears made from the margin of the inhibition zone around a penicillin depot showed a quantitative change of the organisms into long filaments; such filaments were also occasionally seen in ordinary cultures. The micromorphology in the absence or presence of penicillin is given in P1. I, fig. I, 2. For comparison, the normal micromorphology of Moraxella nonliquefaciens, at identical magnification, is shown in Pl. I, fig. 3. Organisms of strain M 2 were distinctly smaller than $M$. nonliquefaciens. They were Gram-negative, with a slight tendency to retention of the first stain. No capsules were observed in Indian ink preparations. The strain was non-motile.

After incubation on blood agar for $20 \mathrm{hr}$, the colonies were I to $\mathrm{I} \cdot 5 \mathrm{~mm}$. in diameter, even, shiny, low hemispherical, and had an entire edge. They were nonpigmented, as confirmed by culture on coagulated serum medium, greyish white, opaque. There was a tendency towards growth into the blood agar, with a resulting slight pitting of the agar surface. The colony consistency was clay-like and coherent; sometimes adherence to the medium was observed. The organisms were not emulsifiable in saline. No haemolysis was seen. There was no anaerobic growth. Slight growth 
occurred at room temperature $\left(20\right.$ to $22^{\circ}$ ). Optimal growth temperature was 33 to $37^{\circ}$. The organisms survived exposure to $45^{\circ}$ for $30 \mathrm{~min}$., but were killed at $56^{\circ}$ in $5 \mathrm{~min}$. The ability to survive on blood agar at room temperature was limited, cultures becoming sterile in 5 to ro days. Growth on nutrient agar was almost as good as growth on blood agar. Elevated carbon dioxide tension enhanced the growth slightly. On simple peptone media such as the nitrate and indole media used, growth was good although somewhat slow. There was also relatively good growth in Hugh \& Leifson medium, I cm. downwards from the surface, without production of acid, but with the appearance of small amounts of a brownish pigment along the stab. The organism grew well in amino acids +biotin medium. No growth occurred in the basal medium with ammonium ions as nitrogen source and glucose, maltose, acetate, citrate or lactate as carbon source, nor in Koser citrate medium. The catalase reaction was weakly positive. Strongly positive oxidase reactions were observed with both the tetramethyl- and the dimethyl- $p$-phenylenediamine reagents. No acid was produced from glucose, maltose, sucrose, lactose, fructose, arabinose, galactose, xylose or mannitol. Nitrate was not reduced to nitrite. Nitrate did not disappear on the addition of $\mathrm{Zn}$ powder. Gelatin and coagulated serum were not liquefied. There was no production of indole or hydrogen sulphide. Urea was not decomposed. There was no oxidative deamination of phenylalanine or tryptophan.

The organism was very sensitive to penicillin $\mathrm{G}$, streptomycin, chloramphenicol, oxytetracycline and erythromycin.

$D N A$ base composition. The buoyant density of DNA of strain M 2 wild type was determined in two different analytical ultracentrifuges and found to be $1.7131 \mathrm{~g} . / \mathrm{cm}^{3}$ and $\mathrm{I} \cdot 7 \mathrm{I} 28 \mathrm{~g} . / \mathrm{cm} .^{3}$, respectively. The DNA gave one very sharp peak, with a gaussian distribution. The average base composition of the DNA, i.e. the mole percentage of guanine + cytosine (mole $\% \mathrm{G}+\mathrm{C}$ ), was calculated from the buoyant density by using the simplified formula: mole $\%(\mathrm{G}+\mathrm{C})=\mathrm{I} 1000 \times(\rho-\mathrm{I} \cdot 660)$, where $\rho=$ buoyant density of DNA (g. $\left./ \mathrm{cm}^{3}\right)$ measured versus the density of Escherichia coli DNA, assumed to be $\mathrm{I} \cdot 7 \mathrm{IO} \mathrm{g} . / \mathrm{cm} .^{3}$ and to contain 50 mole $\%(\mathrm{G}+\mathrm{C})$. This calculation led to 53 mole $\%(\mathrm{G}+\mathrm{C})$ for the strain $\mathrm{M} 2$.

Transformation reactions. The strain $\mathrm{M} 2$ was used as donor and as recipient against each of the strains Neisseria meningitidis B 8I52/66 and N. flava ATCC I422 I in quantitative transformation (short-term DNA exposure). The results are shown in Table I. Ratios of interstrain to autologous transformation of the respective recipients were all in the range from $9 \cdot 2 \times 10^{-3}$ to $\mathrm{I} \cdot 2 \times 10^{-1}$, with the highest ratios observed when the coccal species were used as recipients. The ratios were somewhat higher between strain $\mathrm{M} 2$ and $N$. flava than between strain $\mathrm{M} 2$ and $N$. meningitidis. In a complete additional set of experiments similar to those of Table $\mathrm{I}$ the highest relative variation of ratio value was in the combination of recipient strain M 2 and donor $N$. flava ATCC $\mathrm{I} 422 \mathrm{I}$, which revealed the ratio $\mathrm{I} \cdot 4 \times \mathrm{IO}^{-2}$ instead of $2 \cdot \mathrm{I} \times \mathrm{IO}^{-2}$. By using a dense recipient population and at the same time omitting DNase treatment before plating, plates crowded with 1000 to 10,000 or more heterologous transformant colonies were easily obtained in all these recipient +donor combinations, when at the same time controls without DNA revealed less than Io streptomycin-resistant colonies/ plate. Although assayed at $50 \mu \mathrm{g}$. streptomycin $/ \mathrm{ml}$. the four kinds of heterologous transformants were uniformly able to grow well at $1000 \mu \mathrm{g}$. streptomycin $/ \mathrm{ml}$, as shown by replica-plating to blood agar plates with that concentration of antibiotic. 
Transformant colonies examined microscopically all revealed the morphology (rod or coccus) of the recipient in question.

Semiquantitative transformation experiments (without DNase termination of the transformation process) with highly competent recipients of Moraxella nonliquefaciens NCTC $7784, M$. osloensis A 1920, $N$. catarrhalis NE II and $N$. ovis $199 / 55$, revealed no transformation with the donor strain M2, nor with the donors $N$. meningitidis B I52/ 66, N. meningitidis M 6 and $N$. flava ATCC 14221, which were included for comparison and confirmation of previous data. The results of these experiments are collected in

\section{Table I. Compatibility in streptomycin-resistance transformation between strain $M 2$ and coccal Neisseria species}

In each experiment identical samples of the recipient were exposed simultaneously to the respective donor DNA samples (20 or $40 \mu \mathrm{g}$. $/ \mathrm{ml}$.). Following DNA exposure for $20 \mathrm{~min}$. at $33^{\circ}$, DNase was added and $0 . \mathrm{I} \mathrm{ml}$. spread on each of 2 to 4 plates, which were incubated at $33^{\circ}$ for 5-6 hr. Then streptomycin was added from below the agar and the incubation continued for 3 to 4 days before assay of transformants. The donor mutants had been selected at $500 \mu \mathrm{g}$. streptomycin $/ \mathrm{ml}$.; the transformants were assayed at $50 \mu \mathrm{g} / \mathrm{ml}$. Methods: Bøvre (1964).

Recipient strain; colony count $/ \mathrm{ml}$.

Donor strain

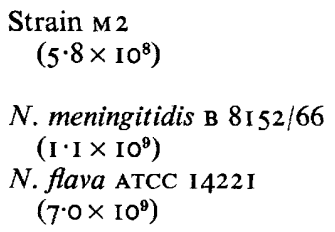

$N$. meningitidis в 8I 52/66
$N$. flava ATCC I422 I
Strain M 2
Strain M 2
$N$. meningitidis в 81 52/66
Strain M 2
$N$. flava ATCC I422 I

N. meningitidis $\mathrm{B} 8152 / 66$

N. flava ATCC 14221

(t)

Strain M 2

N. flava ATCC 1422

ATCC $=$ American Type Culture Collection.

Ratio of interstrain to autologous transformation
$9.2 \times 10^{-3}$ $2 \cdot 1 \times 10^{-2}$
$4 \cdot 9 \times 10^{-2}$
$1 \cdot 2 \times 10^{-1}$

Table 2. The donors $N$. ovis $199 / 55, M$. phenylpyrouvica $2863, M$. nonliquefaciens $4663 / 62$ and $M$. osloensis A 1920 , were chosen as sources of reference transforming DNA because they had low, but still distinct, transforming activities on the respective recipients, as measured in previous short-term experiments where the autologous mutant DNA of the recipients had been included (see legend to Table 2). Using the affinities of the reference donors as parameters in the continuous DNA exposure experiments, it was possible to calculate that the upper limit of any possible transformation between the M 2 donor and the four recipients had to be as low as about $\mathrm{IO}^{-8}$, autologous transformation of each recipient taken as unity (Table 2). This resolution would have been technically impossible by direct comparisons with the high-frequency autologous reaction in continuous DNA exposure, since this procedure did not allow differential dilutions to be made in the parallels to be compared. The rationale for its use was its very high sensitivity in the detection of low transformation activities. A series of semiquantitative experiments (not tabulated) were also made with the strain M2 as recipient and the following donors: $M$. nonliquefaciens 6I 21/68, M. osloensis A 1920, $M$. phenylpyrouvica $2863, M$. kingii $4177 / 66, N$. catarrhalis NE I I, $N$. ovis $199 / 55$. In no case were transformants observed. These experiments were not sensitive as regards very low transformation compatibilities (minimum 
K. BØVRE AND E. HOLTEN

detectable ratio of interstrain to autologous transformation in the order of $10^{-5}$ for the donors of $M$. phenylpyrouvica and $M$. kingii, and in the order of $10^{-4}$ for the other donors).

Table 2. Degree of incompatibility in streptomycin-resistance transformation between strain $M 2$ and Moraxella nonliquefaciens, $M$. osloensis and the 'false neisserias' $(N$. catarrhalis and $N$. ovis)

Methods as described in legend to Table I, except that DNase was not added for termination of DNA exposure (Bøvre, $1967 a$ ) and that incubation times before and after streptomycin addition were 5 to $7 \mathrm{hr}$ and 4 to 5 days.

Recipient strain

M. nonliquefaciens NCTC 7784
M. osloensis A 1920
$=$ ATCC $19976=$ NCTC 10465

N. catarrhalis NE II

N. ovis $199 / 55$
Donor strain

No DNA

N. meningitidis в $8152 / 66$

$N$. flava ATCC I422I

Strain $\mathrm{M} 2$

N. ovis $199 / 55$

No DNA

N. meningitidis B 8152/66

$N$. fava ATCC I422I

Strain м 2

M. phenylpyrouvica 2863

$=$ ATCC $23333=$ NCTC 10526

No DNA

$N$. meningitidis м 6

Strain M 2

M. nonliquefaciens $4663 / 62=$ ATCC I $9975=$ NCTC I0464

\section{No DNA}

$N$. meningitidis м 6

Strain $\mathrm{M} 2$

M. oslosenis A $1920=$ ATCC 19976

$=$ NCTC 10465 .

$\begin{array}{cc}\begin{array}{c}\text { Sum of } \\ \text { resistant } \\ \text { colonies } \\ \text { on } 2 \text { or } 4 \\ \text { plates }\end{array} & \begin{array}{c}\text { Ratio of } \\ \text { interstrain } \\ \text { to autologous } \\ \text { transformation* }\end{array}\end{array}$

0

o

$413<2.5 \times 10^{-5}(a)$

I $\quad<3 \times 10^{-8}$

$\left.\begin{array}{l}0 \\ 0 \\ 1\end{array}\right\} \quad<3 \times 10^{-8}$

$277 \quad 6 \times 10^{-6}(b)$
23
$25<2 \times 10^{-8}$
$\mathrm{I} 8<\mathrm{I} \times \mathrm{IO}^{-8}$
$\begin{array}{rl}18 & <\mathrm{I} \times 10^{-8} \\ 6800 & 6.7 \times 10^{-5}(c)\end{array}$

$<2 \times 10^{-8}$

$<4 \times 10^{-8}$

$2 \times 10^{-5}(d)$

* Autologous transformation = intrastrain transformation of the recipient.

$a, b, c, d$ : Reference donor activities, basis for calculation of ratios in the other parallels. $a, c$ : quantitative determinations (Bøvre, I967a); $b$ : semiquantitative estimate (Bøvre \& Henriksen, $1967 b$ ) with another recipient of $M$. osloensis; $d$ : derived from semiquantitative data of Bøvre, ( 1967 a). Other donors than $\mathbf{2} 2$ and the reference donors were included in parallel for comparison.

ATCC $=$ American Type Culture Collection; NCTC $=$ National Collection of Type Cultures, London.

\section{DISCUSSION}

The micromorphological, cultural and biochemical characters of strain $\mathrm{M} 2$ distinguish this organism from all known moraxellas. The species which has the closest resemblance is Moraxella nonliquefaciens (Bøvre \& Henriksen, 1967a). Strain M 2 differs from $M$. nonliquefaciens by its small dimensions, distinct colony consistency, growth in Hugh \& Leifson medium and in the amino acids + biotin medium (see Baumann et al. I968), and a poor ability to survive on blood agar at room temperature. The strain M2 shows a pattern of affinities in genetic transformation and a $\mathrm{G}+\mathrm{C} \%$ content never observed with oxidase-positive rods. It has no compatibility with known rod-shaped moraxellas (e.g. $M$. nonliquefaciens and $M$. osloensis), nor with the 'false neisserias' (e.g. 
$N$. catarrhalis and $N$. ovis). On the other hand, strain M 2 is distinctly compatible in transformation with the 'true' Neisseria group (e.g. N. meningitidis, N.flava), with which it shares a high $\mathrm{G}+\mathrm{C} \%$ content, unique for an oxidase-positive non-motile rod. If the organism had been a coccus, there would have been no reason to discuss its designation as a Neisseria. Since it is a rod, the relative value of this classically high-weighted character must be considered against molecular composition of DNA and genetic affinities. It is quite possible that cell shape (rod or coccus) may be a genetically more complex character than most single phenotypic traits. However, the very clear difference in terms of $\mathrm{G}+\mathrm{C} \%$ content and the transformation incompatibility (with a 'ribosomal' marker) between strain $\mathrm{M} 2$ and other oxidase-positive rods, taken together with the similarity in $\mathrm{G}+\mathrm{C} \%$ content and genetic compatibility with coccal species, probably characterize the basic relations of strain $\mathbf{m} 2$ better than its shape. We therefore feel that the strain $\mathrm{m} 2$ should be placed in the revised, restricted genus Neisseria (Henriksen \& Bøvre, 1968). The alternative construction of a new genus for the organism is not favoured, because of the relatively high genetic affinity to Neisseria species. However, since most of the studies of heterologous transformation in genus Neisseria were made with another technique than the one used here (Catlin \& Cunningham, I96I), we hesitate to draw firm conclusions about the exact degree of high compatibility between strain M 2 and the coccal species of the genus, as compared with the mutual relations of the latter. Since there is no known case of genetically stable intraspecies variation from coccus to rod or vice versa, we consider it advisable to give the strain M 2 rank as a separate species without testing the genetic affinities to all species within genus Neisseria.

We propose the name Neisseria elongata for the new species, the specific epithet expressing the rod shape, distinguishing it from all other known members of genus Neisseria. The strain M 2, the only isolate as yet, is proposed as the type strain. It has been deposited in the National Collection of Type Cultures, London, and in the American Type Culture Collection, as NCTC I0660 and ATCC 25295.

As a consequence of the existence of Neisseria elongata, the revised definition of the Family Neisseriaceae Prévot, 1933, Genus I, Neisseria Trevisan, I885, as proposed by Henriksen \& Bøvre (I968), should be emended by changing the first sentence to 'Organisms coccal or rod-shaped'. That this family now appears to contain two genera, each with coccal and rod-shaped species (loc. cit.), may warrant a general reconsideration of cellular shape as a master criterion in bacterial classification at genus and higher level.

A condensed description of Neisseria elongata follows.

\section{Description of Neisseria elongata sp.nov.}

Micromorphology. Small slender rods, often occurring as diplobacilli or in short chains. Some organisms may be coccoid, which change uniformly into long threadlike filaments by culture in the presence of low concentrations of penicillin. Gramnegative, with some tendency to resist decolorization. Not encapsulated. Non-motile.

Colonies. Greyish white, opaque, I to I $5 \mathrm{~mm}$. in diameter after incubation of blood agar cultures for $20 \mathrm{hr}$. Even, shiny, low-hemispherical with an entire edge. Clay-like, coherent consistency. Some pitting of the agar. No haemolysis.

Relation to oxygen. Aerobe. 
Temperature. Slight growth at 20 to $22^{\circ}$; optimal growth at 33 to $37^{\circ}$, survives $45^{\circ}$ for 30 min., but is killed at $56^{\circ}$ in $5 \mathrm{~min}$.

Viability. Blood agar cultures are not viable after 5 to ro days at 20 to $22^{\circ}$.

Growth requirements. Growth on nutrient agar almost as good as on blood agar. Good but slow growth in peptone media. Growth in Hugh \& Leifson medium in the upper $\mathrm{I} \mathrm{cm}$. Growth in amino acids + biotin medium. No growth in basal medium with ammonium ions as nitrogen source and glucose, maltose, acetate, citrate or lactate as carbon source.

Biochemical reactions. Weakly positive catalase reaction. Oxidase reaction strongly positive with tetramethyl- and dimethyl-p-phenylenediamine reagents. No acid produced from glucose, maltose, sucrose, lactose, fructose, arabinose, galactose, xylose or mannitol. Nitrate not reduced to nitrite. Gelatin and coagulated serum not liquefied. No production of indole or hydrogen sulphide. No decomposition of urea. No oxidative deamination of phenylalanine or tryptophan.

Antibiotic sensitivity. Highly sensitive to penicillin G, streptomycin, chloramphenicol, oxytetracycline and erythromycin.

Habitat. Human nasopharynx.

Pathogenicity. Unknown.

DNA base composition. One strain: 53 mole \% guanine + cytosine, as calculated from buoyant density in $\mathrm{CsCl}$ gradient $\left(1 \cdot 7 \mathrm{I} 3 \mathrm{~g} . / \mathrm{cm} .^{3}\right)$.

Genetic affinities. Incompatible in streptomycin-resistance transformation with species of genus Moraxella, as defined by Henriksen \& Bøvre (1968). Compatible with members of genus Neisseria (loc. cit.), with ratios of interstrain to autologous transformation in the order of $0.0 \mathrm{I}$ to $\mathrm{O} \cdot \mathrm{I}$.

We are most grateful to Dr S. D. Henriksen for valuable discussions and advice We are indebted to $\mathrm{Mr} \mathrm{M}$. Fiandt for expert assistance in making the analytical centrifuge runs and to Dr W. Szybalski for allowing this work to be done in the McArdle Laboratory, University of Wisconsin. Thanks are due to Mrs Valeria E. A. Gencsy Gacek for technical assistance.

\section{REFERENCES}

Baumann, P., Doudoroff, M. \& Stanier, R. Y. (I968). Study of the Moraxella group. I. Genus Moraxella and the Neisseria catarrhalis group. Journal of Bacteriology 95, 58.

Beno, D. W., Devine, L. F. \& Larson, G. L. (I968). Identification of Neisseria meningitidis carbohydrate fermentation pattern in Mueller-Hinton broth. Journal of Bacteriology 96, 563.

BøvRE, K. (1964). Studies on transformation in Moraxella and organisms assumed to be related to Moraxella. I. A method for quantitative transformation in Moraxella and Neisseria, with streptomycin resistance as the genetic marker. Acta Pathologica et Microbiologica Scandinavica 6r, 457.

BøvRE, K. (1965). Studies on transformation in Moraxella and organisms assumed to be related to Moraxella. 4. Streptomycin resistance transformation between asaccharolytic Neisseria strains. Acta Pathologica et Microbiologica Scandinavica 64, 229.

BøvRE, K. (1967a). Studies on transformation in Moraxella and organisms assumed to be related to Moraxella. 7. Affinities between oxidase positive rods and neisseriae, as compared with group interactions on both sides. Acta Pathologica et Microbiologica Scandinavica 69, 92.

BøVRE, K. (1967b). Transformation and DNA base composition in taxonomy, with special reference to recent studies in Moraxella and Neisseria. Acta Pathologica et Microbiologica Scandinavica 69, I 23.

BøVRE, K. (1969). Identification of an asaccharolytic Neisseria strain causing meningitis. Acta Pathologica et Microbiologica Scandinavica 76, 148. 


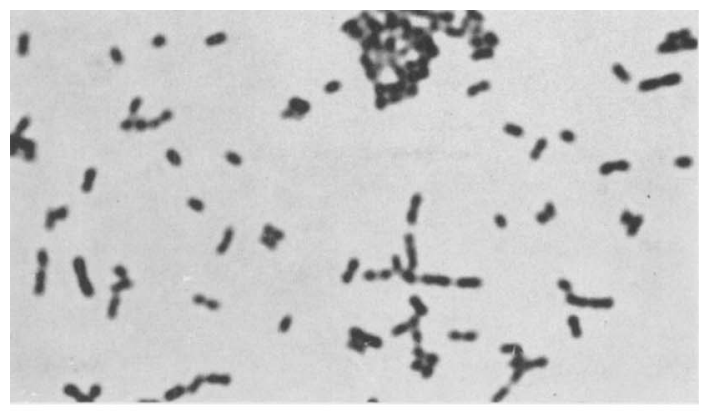

Fig. I

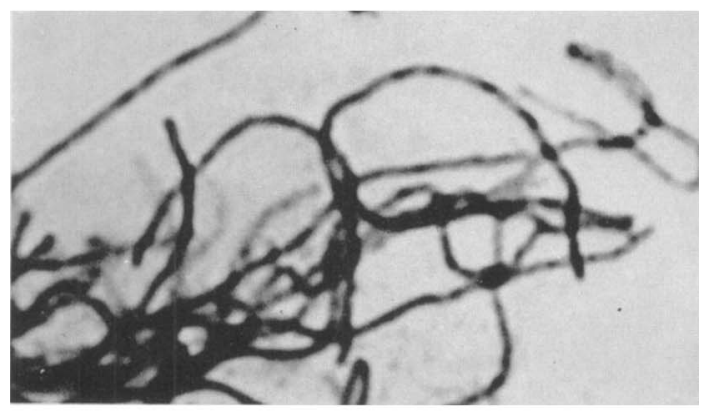

Fig. 2

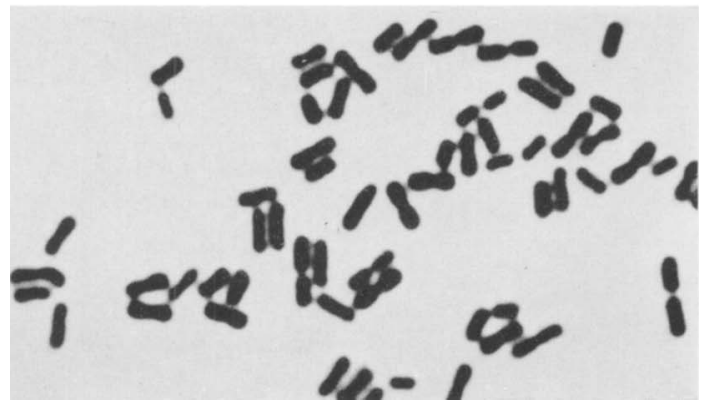

Fig. 3 
Bøvre, K., Fiandt, M. \& Szybalski, W. (1969). DNA base composition of Neisseria, Moraxella and Acinetobacter, as determined by measurement of buoyant density in $\mathrm{CsCl}$ gradients. Canadian Journal of Microbiology 15, 335.

Bøvre, K. \& Henriksen, S. D. (1962). An approach to transformation studies in Moraxella. Acta Pathologica et Microbiologica Scandinavica 56, 223.

Bøvre, K. \& Henriksen, S. D. (1967a). A new Moraxella species, Moraxella osloensis, and a revised description of Moraxella nonliquefaciens. International Journal of Systematic Bacteriology 17, 127.

BøVRe, K. \& Henriksen, S. D. (1967b). A revised description of Moraxella polymorpha Flamm 1957, with a proposal of a new name, Moraxella phenylpyrouvica, for this species. International Journal of Systematic Bacteriology 17, 343.

Catlin, B. W. \& Cunningham, L. S. (196I). Transforming activities and base contents of deoxyribonucleate preparations from various neisseriae. Journal of General Microbiology 26, 303.

Henriksen, S. D. \& Bøvre, K. (I968). The taxonomy of the genera Moraxella and Neisseria. Journal of General Microbiology 5r, 387.

Henriksen, S. D. \& BøVre, K. (I969). Corroding and spreading colonies in Moraxella nonliquefaciens. Acta Pathologica et Microbiologica Scandinavica 76, 459.

JysSUM, K. (1965). Isolation of auxotrophs from Neisseria meningitidis. Acta Pathologica et Microbiologica Scandinavica 63, 435.

JysSUM, K. \& LIE, S. (1965). Genetic factors determining competence in transformation of Neisseria meningitidis. I. A permanent loss of competence. Acta Pathologica et Microbiologica Scandinavica 63, 306.

\section{EXPLANATION OF PLATE}

Fig. I. Neisseria elongata, strain м 2 (type strain). Blood agar culture. Stained with Loeffler methylene blue. $\times 2300$.

Fig. 2. Same strain as in Fig. I after growth on blood agar with low penicillin concentration. Stained with Loeffler methylene blue. $\times 2300$.

Fig. 3. Moraxella nonliquefaciens $4663 / 62=$ ATCC $19975=$ NCTC I0464. Blood agar culture. Stained with Loeffler methylene blue. $\times 2300$. 